\title{
Potential Hazards and Possible Safety Precautions in Electrotherapy
}

\author{
Maruf, F.A., Umunnah, J.O., Akosile, C.O. \\ Department of Medical Rehabilitation, College of Health Sciences and Technology, Nnamdi Azikiwe University, \\ Nnewi Campus, Nnewi, Anambra State, Nigeria \\ Correspondence \\ F.A. Maruf, Department of Medical Rehabilitation, College of Health Sciences and Technology, Nnamdi \\ Azikiwe University, Nnewi Campus, Nnewi, Anambra State, Nigeria・mafaad@yahoo.com
}

\begin{abstract}
SUMMARY
The use of various electrotherapeutic modalities in physiotherapy is known to offer beneficial effects for patients for whom the modality has been found to be clinically suitable. However, there are a few grey areas that need to be addressed in order to maximize the benefits derived from the use of these modalities. Though these modalities have direct benefits for the patients, the physiotherapist, support staff and students, and even the patient are exposed to some danger from, or related to, their use, unless certain safety precautions are instituted. This article presents the potential dangers associated with different electrotherapeutic modalities and the possible preventive measures that could be undertaken to protect individuals likely to be exposed to these dangers.
\end{abstract}

KEYWORDS: hazards, safety, precaution, electrotherapy

\section{INTRODUCTION}

Evidence exists on both local and general side effects of electrotherapy (Partridge and Kitchen, 1999). In a study on adverse effects of electrotherapy among patients from 148 hospitals in England and Wales, Partridge and Kitchen (1999) reported 98 local effects such as burns, skin rashes and pain and 87 general effects such as nausea and fainting. A number of different agents were implicated, but the largest number of reports was related to the use of interferential. Between March 1991 and April 1992, a total of 51 clinical reports on electrotherapy were received from various physiotherapy clinics: interferential (20), pulsed short wave diathermy (PSWD) (15), transcutaneous nerve stimulation (TNS) (8), ultrasound (3), ultraviolet radiation (UVR) (3), and LASER (2) (Partridge, 1992). Some of the reported effects were general, such as feeling unwell after interferential treatment or nausea with TNS, PSWD, interferential, UVR, or LASER treatments. An increase in pain was reported with interferential, PSWD, TNS, or ultrasound. Skin problems of different types were mentioned in relation to most modalities and oedema specifically for interferential, PSWD and TNS (Partridge, 1992). An important problem area identified was that of exacerbation of already existing conditions including eczema, Maniere's disease, and pancreatic symptoms in a patient with a history of pancreatitis (Partridge, 1992). There was also a report of a graft wound, which opened widely after treatment with PSWD (Partridge, 1992). The reason for the adverse effects may occasionally be related to machines delivering higher than indicated doses (Partridge and Kitchen, 1999), or due to inappropriate techniques of application. Also, a sudden or undetected electrical fault is a potential source of hazard to both the patient and the physiotherapist.

\section{Electrical Safety}

Electrocution is a serious possible hazard with electrotherapy. It has a lot of causative factors which include faulty exposure of the conducting cable of the machine, lack of or improper earthing of equipment, 
improper circuit connection, faults in an appliance and so on (Fox et al., 2007). All these factors are preventable unless they develop suddenly. Improper circuit connection is usually not a problem when equipment is new. However, this does not mean that new equipment cannot come with a circuit connection problem. Most circuit connection problems start after the machine develops a fault and is taken for repair; in the process, the normal circuit connection may be tampered with. This is where a great deal of caution is needed. Adequate technical inspection is necessary to forestall these potential electrical hazards; these range from visual inspection of a cord grip on a plug to the detailed testing of a major electrical installation, which may require an expert (Allison, 1993). The frequency of inspection of an equipment should depend on the type of equipment, whether it is fixed or portable, the manner in which it is used, and the frequency of use (Allison, 1993). Also, the physiotherapist must check all electrotherapy equipment for wear and tear of leads, sockets, plugs, electrodes, connections, wire insulation, indicator lights, dials, switches, and control (Fox et al., 2007; George, 2007).

The problem of cable exposure can be conveniently forestalled by making sure that all electrical appliances come with a durable and protective rubber insulator, and that, from time to time, the cable is examined for any form of defect. Proper earthing of the circuit can be ensured by the use of a three-pin plug (Fox et al., 2007). Fault in an equipment can be prevented through regular and thorough servicing (Fox et al., 2007).

In order to ascertain the safety of electrotherapy equipment, general requirements for the safety of medical electrical equipment should be confirmed from the manufacturer, either by the supplier or, preferably, in the hospital or clinic, by suitably trained hospital physicists or engineers (BS5724, 1989) before any piece of equipment is put to use. The physiotherapist should ensure that all portable electrical appliances are maintained and stored away safely to avoid them being stepped on or kicked by people moving about (EWR, 1989). Also, there must be a first-aid kit on site under the control of a responsible person (HS-First Aid, 1981) in case of any unavoidable accident.

\section{THERMOTHERAPY}

\section{Superficial Heating Modalities}

The main problem with superficial heating modalities has to do with the patient. The development of burns or scalds in patients depends on the equipment in question. Very short distance between the patient and the infra-red radiation (IRR) lamp and use of insufficient layers or thickness of towels in hot pack therapy can result in burns and scalds respectively (Kitchen, 2002). Heat treatment around areas contraindicated in heating modalities, such as devitalized tissue or areas with metal implants, can also cause burns or gangrene. In the case of modalities like the hot pack and some IRR lamps, where intensity regulation may not be available, the amount of heat received by a patient will be determined by the layers or thickness of towels separating the body part and the pack and the distance of the lamp from the part being treated (CPAH, 2008) respectively. The distance and thickness should be such that erythema does not occur until ten minutes after the treatment has started, while the application time should be short and under no circumstance must it exceed 30 minutes (CPAH, 2008). Infra-red radiation lamps which have output power indicators, usually referred to as the electrical power consumed by the lamp or heater (Low et al., 1992), could be used in conjunction with distance setting and subjective feedback from the patient.

Besides the regulation of intensity, time and distance between the machine and the part being treated, loss of skin sensation may cause burns, especially in cases where the patient is not able to indicate the level of heat he/she perceives. Also, the patient should be instructed not to touch the glass bulb inside the lamp while in use to avoid burns. The patient's skin must be clean and free of grease or liniment and the eyes protected with goggles or cotton wool soaked in water, especially if any part of the face is to be irradiated (CPAH, 2008). In essence, these parameters should be carefully regulated using the standardized recommended prescriptions and dosages as guides. All these go a long way in ensuring that undue heat exposure is not experienced by the patient, and that sensitive parts of the body are protected.

Lasers are classified into four groups according to the level of hazard they present, with class 1 being the least and class 4 the most hazardous (CPAH, 2008). The hazard includes damage to non-targets, such as sebaceous glands (CPAH, 2008). Lasers are contraindicated in cancerous tissue, unclosed fontanelle of infants, over the pregnant uterus, the heart of patients with pacemakers, as well as areas of venous thrombosis, phlebitis and arterial disease (CPAH, 2008). Also, the eyes of both the physiotherapist and the patient must be protected with suitable goggles provided for the specific type of laser in use (CPAH, 2008). 
In addition to the use of eye protectors, treatment in a brightly lit area is recommended to ensure constriction of the pupil and thus reduction in the amount of radiation that enters the eye (Fox et al, 2007; CPAH, 2008). Shining a laser beam into the air, even by accidental reflection or diffuse scattering, must be avoided. This is ensured by switching on the laser beam only when the applicator is in contact with the skin. If the applicator is used on infected or contaminated skin, it must be cleaned and disinfected with a suitable disinfectant solution afterwards (CPAH, 2008). Also, the physiotherapist must check the laser probes for output. This will depend on the probe and machine used (George, 2007).

Generally, time factor as a means of preventing heat overdose applies to all equipment in electrotherapy. Other modalities such as wax therapy and cryotherapy have their own special ways of preventing burns in patients. For wax therapy, the wax bath has a thermostat that regulates the temperature of the wax. Furthermore, use of pure paraffin wax ensures that the temperature of the wax ranges between 45 and 49 degree celsius (George, 2007). In a situation where a wax bath does not come with a thermostat, a thermometer can be used to ascertain the temperature prior to application to the skin (George, 2007). Also, the sterilizing procedure for wax must be followed, at least once a week, or as indicated in the manufacturer's manual. Where self-cleaning is not possible, the used wax should be replaced with clean wax (George, 2007). It is important to know that wax is a highly flammable substance, and hence should be kept away from naked fire or even embers (Fox et al., 2007). Above all, adequate explanation of the treatment in terms of the level of heat to be felt is highly precautional (Low et al, 1992). Furthermore, inspection as well as assessment of the area to be treated for skin sensation should be routinely carried out.

\section{DEEP HEATING MODALITIES}

Short wave and microwave diathermy units heat tissue through absorption of energy from electromagnetic fields electric and magnetic fields. The nature of these fields make it impossible to concentrate all the energy in the tissue being treated, and anyone standing close to diathermy equipment, for example the physiotherapist, absorbs a small amount of electromagnetic energy (Martin et al, 1990). The fields associated with capacitive treatments are generally more than those with inductive applicators. Furthermore, the highest electric field strengths occur near the electrodes, while magnetic fields are greatest around the cable (Martin et al, 1990). Both the inductive and the capacitive procedures involve production of the two fields. However, while both fields are utilized for treatment in the inductive method, only the electric field is utilized in the capacitive procedure. Even with the diffuse nature of energy, the presence of metal implants in the tissue can cause burns due to the concentration of adjacent field lines produced by shortwaves or microwaves. The International Radiation Protection Association has issued guidelines regarding electromagnetic radiation based on thermal effects (IRPA/INIRC, 1988), and these have been adopted by the National Radiological Protection Board (NRPB, 1989) for implementation in the UK. Those guidelines which are relevant to the use of diathermy equipment are as follows:

- The average absorption rate in the body over any sixminute period should not exceed $0.4 \mathrm{~W} / \mathrm{kg}$ for workers.

The maximum energy absorption rate for workers in any $0.1 \mathrm{~kg}$ of tissue of the hand, wrists, feet and ankle should not exceed $20 \mathrm{~W} / \mathrm{kg}$, or $10 \mathrm{~W} / \mathrm{kg}$ in any other tissues.

- Maximum electric field strength should not exceed 61 $\mathrm{V} / \mathrm{m}$.

- Maximum mean magnetic field strength should not exceed $0.16 \mathrm{~A} / \mathrm{m}$.

- Maximum mean power density should not exceed 10 $\mathrm{W} / \mathrm{m}^{2}$.

Peak field strength should be no greater than 32 times the limits for mean field strengths; peak power density should be no greater than 1,000 times the limit for mean power density.

\section{Continuous Wave Equipment}

Observation of working practices has shown that physiotherapists spend most of their time at distances greater than 1 metre from the electrodes and cables of a diathermy unit (Martin et al, 1990). If the physiotherapist maintains this distance during treatments, and avoids approaching within 0.5 metres, even for a short period, when the field is switched on, there is little danger of exposure exceeding the reference levels. However, particular care should be taken when higher power settings are used. Positioning of electrodes in such a way that the operator does not have to walk past the electrodes in order 
to reach the control panel will help to reduce exposure (Martin et al, 1991).

The level of exposure of the physiotherapist's eyes is small but care should be taken to ensure that the patient's eyes are not closer to the electrodes and cables than necessary during treatment. Fields within 0.2 metres of the electrodes could potentially produce specific absorption rates (SARs) similar to those at which ocular effects have occurred in microwave therapy, although no such effects have been reported with short wave fields. However, recent research suggests that with modern shortwave diathermy (SWD) machines, field strengths beyond 1 metre should fall below the safe exposure limits (Martin et al, 1990; Martin et al, 1991; McDowell and Lunt, 1992). One metre is therefore the minimum recommended distance between a SWD machine and personnel, patient or other electrotherapy equipment. This ' 1 meter' recommendation applies anywhere treatment is carried out (Kirnen, 1992). In departments where patients are treated with continuous wave diathermy in adjacent cubicles or rooms, a space of at least a metre should be left between cubicles, and between couches, where possible, so that other patients and physiotherapists are at least 1 metre from the equipment, when the field is on (Martin et al, 1991; Kirnen, 1992). Moreover, other electrotherapy devices, especially electrical stimulation apparatus should be kept at least 2 meters from the PSWD machine. When operating two diathermy machines simultaneously, they should be positioned at least 3 metres apart (CSP-ERUS, 1997).

\section{Pulsed Shortwave Diathermy (PSWD)}

For pulsed diathermy equipment, electric and magnetic field strengths above the derived reference levels extend to 0.3 to 0.5 metres from the electrode of the Curapuls and Erbotherm units operated in capacitive modes with low or medium pulse settings. The highest pulse and power settings could extend to 0.8 metres. However, with inductive application, field strengths above the limit rarely extend more than 0.2 metres from the electrode or cable. The physiotherapist and all other personnel are therefore advised to stand at a distance of at least 0.5 metres from the electrodes and cables when the units are used in capacitive modes at low or medium power settings, and 0.3 metres when single inductive electrodes are used (Martin et al, 1991). However, CSP-ERUS (1997) recommends a distance of 1 metre from the operating unit and the operator. Therefore, by implication, pregnant physiotherapists may ask a colleague to turn the machine on while the machine turns off automatically at the expiration of the set duration.

\section{SONOTHERAPY [Ultrasound Sound]}

Ultrasound (US) is a form of mechanical energy produced at frequencies above the range of human hearing (approximately $20 \mathrm{KHz}$ ). Treatment can be given in either continuous or pulsed mode, the latter typically involves pulsing regimes of either 1,2 or 5 followed by either 1, 2, 5, or 8 minutes off (SEEWG, 1990).

A number of surveys on the output of therapeutic ultrasound equipment (Reacholi and Benwell, 1979; Rivest et al, 1986) have revealed discrepancies between the indicated and actual ultrasonic output of many devices (SEEWG, 1990). In the interests of safety and effectiveness, it has been recommended that the output of all therapeutic ultrasound equipment be calibrated by suitably trained personnel on delivery and at least once a month (Rivest et al, 1986; Guidelines for the Safe Use of Ultrasound, 1989) to ensure that the ultrasonic power, as measured by a power metre (such as a radiation balance), is indicated with an accuracy of $\pm 20 \%$ (BS5724, 1985). The intensity and pulsing regimen accuracy should be checked at least annually by a suitably trained physicist or engineer using, in the case of pulsing regimen accuracy, a pressure-sensitive detector (hydrophone) and oscilloscope (BS5724, 1985). The effective radiating area of each applicator should be provided by the manufacturer, so that the intensity can be recorded for each treatment. The maximum spatial average and temporal peak intensity available should not exceed $3.0 \mathrm{~W} / \mathrm{cm}^{-2}$, since higher intensities may be painful or damaging (Dyson, 1987). The effective radiating area can be determined by scanning the ultrasound field with a hydrophone at a constant distance of, for example, $5 \mathrm{~mm}$ in front of the applicator face while it is submerged in degassed water, and calculating the area at which the output is greater than $5 \%$ of the spatial maximum at any point in the ultrasonic field at this distance (BS5724, 1985).

Standing waves is a major cause of burns in sonotherapy. It occurs as a result of a combination of incident and reflected rays. To reduce the likelihood of standing waves, which can damage tissue and may also lead to thrombus formation, the treatment head must be moved continuously across the treatment area (CPAH, 2008). 
Formation of standing waves can also be prevented by ensuring that no part of the operator's body is in the path of the ultrasound emitted from the applicator, especially, when the ultrasound is being applied to the patient via a water bath (SEEWG, 1990). In such circumstances, if the operator's hand has to be immersed in the bath while the applicator is active, care should be taken to minimize exposure to any reflected or scattered ultrasound. This can be done by wearing a dry, knitted glove inside a waterproof rubber or plastic glove to provide a protective air-filled gap, across which ultrasound cannot travel (SEEWG, 1990).

Transient cavitation is another source of burns caused by the pressure changes applied to the tissues by the sound waves. It occurs as a result of collapse of a bubble of gas formed as a result of a mechanical effect (Forster and Palastanga, 1985). This can be prevented by using intensities of about $3 \mathrm{watt} / \mathrm{cm}^{2}$ (Hill and Ter Haar, 1981; The International Electrical Commission, 1984), using a pulsed source of ultrasound, or moving the treatment head during insonation. Above all, ultrasound should not be used if contraindicated (SEEWG, 1990).

\section{ACTINOTHERAPY [Ultraviolet Radiation]}

Ultraviolet radiation (UVR) is used by physiotherapists to treat a number of conditions, particularly skin diseases such as psoriasis and acne. The common lamps used, such as the Alpine sunlamp, emit high levels of UV-B (290-320nm) and UVC (200-290nm) radiation which can produce harmful acute effects in the eyes and on the skin. UVA is generally considered the least harmful, but is not totally safe (Wood and Reed, 1990). According to Forster and Palastanga (1985), UVA is not beyond suspicion as a carcinogenic agent. Furthermore, the radiation produced by many types of lamps is not well-contained and measurements have indicated that ultraviolet exposure hazard exists in the vicinity of these lamps, from direct radiation beams and from scattered and reflected UVR (Diffey and Lanley, 1986).

Much of the literature concerning the danger of UVR discusses the effects of prolonged exposure to natural sunlight. To some extent, this information may also be of importance in the clinical environment as cumulative exposure to UVR over a number of years should be considered (Kitchen and Partridge, 1991). In fact, Schothorst et al (1985) demonstrated that long-term psoriatic patients were subjected to higher UVR exposure levels than outdoor workers in the Netherlands. A rough estimation of the annual exposure in this way by indoor workers in the UK is equivalent to about 100 times the minimal erythema dose (MED) - an exposure of UVR which results in just-perceptible reddening of the skin 24 hours after exposure and is a measure used by photobiologists to indicate the biologically-damaging effect of radiation (Low, 1986).

It is well known that repeated and prolonged exposure to UVR, particularly to UVB, over many years can result in chronic degenerative changes in the skin and the development of premalignant and malignant skin lesions (Urbach, 1982). Tring (1981) suggests that UVB is the band that induces skin cancer in laboratory animals. He also suggested that there is epidemiological evidence that UVB causes skin cancer in man illustrated by a higher incidence of skin cancer among young Caucasians in Australia. Forster and Palastanga (1985) and Tring (1981) indicated that UVB is also responsible for producing other changes in the human skin, such as solar elastosis or ageing. Sliney (1986) reported UVB as producing such effects as skin cancer, sunburn (erythema), a form of snow blindness (photokeratitis), and the production of cataracts in laboratory animals. UVC is considered to be germicidal in nature causing destruction of normal skin (Wood and Reed, 1990).

Swanbeck (1984) lists the hazards of UVR as both a short-term risk of burning (erythema, blistering, and pain) and a long-term risk of actinic elastosis, or wrinkling of the skin, and carcinogenesis. Faber (1986) expanded on these dangers, and added the danger of damage to the eyes, including photokeratitis, conjunctivitis, and possibly some forms of cataract. These usually occur from overexposure to all types of UV lights (CPAH, 2008).

An increase in non-melanoma skin cancer and squamous cell cancer of the male genitalia of patients treated with UVA and psoralen-based drugs (PUVA) has been noted (Young et al, 1982). PUVA treatment may also exacerbate the effects of previous exposure to carcinogens. These adverse effects could be minimized by the use of low doses and minimal exposure area (Burns, 1989).

Apart from dose, frequency of application is also a key factor. Epidemiological studies have shown that for a group of subjects with a given genetic susceptibility, age and annual ultraviolet exposure are the two most important factors in determining the relative risk of non-melanoma skin cancer. Expressed mathematically the risk is 
proportional to annual UV $\operatorname{dose}^{2} \mathrm{x}$ age $\mathrm{e}^{5}$ (Fears et al, 1977). According to Forbes et al (1979), low daily doses produce carcinoma at a lower cumulative dose compared to higher but less frequent doses. Since the carcinogenic effects of UVR coincide with erythematous effects in terms of wave length (Green et al, 1988), a spectrally specific lamp which minimizes erythematous effects may, therefore, be helpful in avoiding possible cellular changes (Kitchen and Partridge, 1991). Also, first-time exposure must be limited to the minimum time and only increased gradually on subsequent exposures, provided no adverse reaction is experienced (CPAH, 2008). Typically, not more than 20 sessions per year is recommended.

Ultraviolet radiation is not advisable in individuals with fair and hypersensitive skin, lots of freckles or moles often associated with red hair, a family history of skin cancer, pregnant women, herpes simplex infection as well as patients on drugs causing photosensitivity, such as tetracycline (CPAH, 2008). Those with contact lenses should have them removed while goggles should be worn by both the physiotherapist and the patient. Cotton wool pads or sunglasses provide insufficient protection from reflected radiation. Furthermore, creams, perfumes or cosmetics must not be used or should be cleaned off before receiving or giving UVR (CPAH, 2008).

\section{DIRECT CURRENT (DC)}

Direct current (DC) also known as electromotor stimulation (EMS) is electrical current that flows for one second or longer. Direct current is categorized into two: pulsated interrupted direct current and low frequency continuous direct current or galvanic current (GC) (CPAH, 2008). The galvanic machine produces direct current, which travels through the skin across two electrodes, and produces a chemical effect within the skin (George, 2007). Unlike pulsated DC, in which the effects produced in the body is immediately neutralized by the interruption of flow of pulse during the off time, GC produces hazards which include electrocution, local infection, chemical and heat burns as well as powerful and prolonged muscle contraction which can damage the heart muscles and stop circulation. There is also the risk of tissue reaction or skin infection from the electrodes (Charman, 1990). There may also be undue localization of current due to open wounds or skin lesions, such as eczema (CPAH, 2008). Also, current could provoke undesirable metabolic activity in cancerous tissue or in healed tuberculous infection. Current which is not biphasic may lead to skin damage or irritation, especially if there is loss of sensation (CPAH, 2008).

General precautions for GC include prevention of strong muscle contraction, which can cause joint or muscle damage, detachment of thrombus, spread of infection, and haemorrhage; prevention of stimulation of autonomic nerves that may cause altered cardiac rhythm or other autonomic effects (CPAH, 2008). Also, the current must not change in intensity too rapidly or the pain receptors of the skin may be stimulated, in addition to the muscle. To ensure safety, electrodes must stay in contact with the skin while the machine is operating and the intensity of the current should be reduced when bony regions of the face are being treated (CPAH, 2008). To avoid tissue reaction, silver or platinum electrodes should be used (Charman, 1990).

Between treatments, the electrode pads and the securing straps must be disinfected in hypochlorite solution, and thoroughly rinsed (George, 2007). The electrode pads should be strapped securely, and evenly soaked with the electrode solution to prevent local galvanic burns due to high alkalinity (CPAH, 2008). The two electrodes should not be allowed to come in contact while the machine is on to avoid short circuiting, and the patient's skin should be washed immediately after treatments to remove the alkaline solution produced by the treatment which will soften the skin and cause redness in the vicinity of the electrode (CPAH, 2008). Electromotor stimulation equipment must be checked thoroughly by the designated person, using an oscilloscope, before it is used for treatment. At every review appointment, a visual inspection of EMS equipment must be made. If the patient reports a problem with the functioning of the stimulator, further checks must be made (George, 2007).

\section{ALTERNATING CURRENT}

A faradic current generator is a single transistor relaxation oscillator which has an adjustable firing rate. Faradic current (FC) is produced by conversion of rapidly interrupted direct current into alternating current, which is applied to the body by means of electrodes. This direct application of current along with its potential fluctuations sometimes causes unpleasant shock in subjects. Over time, an improved form of FC has been produced to minimize this hazard. However, a malfunction could still expose 
subjects to unexpected shocks as the current is basically received directly from the transformer (USP 4124030). Faradic current is capable of producing a mechanical effect without a chemical reaction (Quick Acne Remedy). To ensure comfort in the use of FC, the frequency should be graded such that even when the purpose of treatment is achieved, there is no pain (USP 4124030).

\section{CONCLUSION}

Safety precautions such as avoiding contraindications to individual modalities, ensuring electrical safety of the equipment, giving comprehensive instructions, and maintaining adequate distance between the body parts and the equipment as well as delivering optimal dosage of the modalities to the patient are crucial to ensuring safety in electrotherapy. Above all, employers, and paid and selfemployed physiotherapists need to ensure the health, safety and welfare of persons attending their clinics.

\section{References}

Allison, R. 1993. Putting electrical safety in perspective. Physiotherapy 79: 471.

AMREX-ZETRON Inc. 2000. Electrotherapy equipment. www.amrex-zetron.com

British Standards Institution. 1989. BS5724: Safety of Medical Electrical Equipment: Part 1. General Requirements; British Standards Institution, London, England

British Standards Institution. 1989. BS5724: Section 2.5:1985, British Standard Medical Electrical Equipment. Part 2. Particular requirements for safety. Section 2.5 Specification for safety of ultrasonic therapy equipment. BSI, London.

Burns, F. 1989. Cancer risks associated with therapeutic irradiation of the skin. Archives of Dermatology 125: 979-981.

Charman, R.A. 1990. Exogenous currents and fields: Experimental and clinical applications. Physiotherapy 76(12): 743-750.

Code of Practice for Application of Heat (CPAH). 2008. General Precautional Guidelines for Electrotherapy.

www.thurrock.gov.uk/licencing/pdf

Diffey, B.L. and Langley, F.C. 1986. Evaluation of Ultraviolet Radiation Hazards in Hospitals, Report 49, Institute of Physical Sciences in Medicine, London.

Dyson, M. 1987. Mechanism involved in therapeutic ultrasound. Physiotherapy 73(3):116-120.

Dyson, M. 1989. The use of ultrasound in sports physiotherapy. In: Grisomo, V. (ed) Sport Injuries: International perspective in physiotherapy. Churchill, Livingstone, Edinburgh

Faber, M. 1989. Ultraviolet radiation. In: Suess, M. and Benwell-
Morison, D. (eds). Non-ionizing Radiation Protection ( $2^{\text {nd }}$ ed.), WHO Regional Publications, European Series, no 25, Geneva.

Fears, T.R., Scotto, J. and Schneiderman, M.A. 1977. Mathematical models of age and ultraviolet effects on the incidence of skin cancer among whites in the United States. American Journal of Epidemiology 105: 420-427.

Forbes, P.D., Davies, R.E., Urbach, F. 1979. Ageing, environmental influences influences and carcinogenesis. Journal of Investigative Dermatology 73: 131-134.

Forster, A. and Palastanga, N. 1985. Clayton's ElectrotherapyTheory and Practice, $9^{\text {th }}$ ed. Bailliere Tindall, London.

Fox, J. and Sharp, T. 2007. Practical Electrotherapy: A Guide to Safe Application. Churchill Livingstone (Elsevier), Philadelphia.

Georges, S. 2007. Policies and guidance for the use of electrotherapy by physiotherapists. www.wandsworth pct.nhs.uk/policies

Guidelines for the Safe Use of Ultrasound. Part 1. Medical and Paramedical Applications. Safety Code 231989. Environmental Health Directorate, Health Protection Branch, Canadian Government Publish Center, Ottawa, Canada.

Health and Safety (First Aid) Regulation (HS-First Aid). 1981.

Hill, C.R. and Ter Haar. 1981. Ultrasound and non-ionising radiation protection. In: Suess M. and Benwell-Morison D. (eds). Non-ionizing Radiation Protection ( $2^{\text {nd }}$ ed.), WHO Regional Publications, European Series, no 25, Geneva.

Regional Publication, European Series No 10, World Health Organization, Copenhagen.

International Electrical Commission. 1984. Medical Electrical Equipment, Part 2: Particular Requirement for the Safety of Ultrasonic Therapy Equipment, Publication 601-2-5.

International Radiation Protection Association/ International NonIonising Radiation Committee. 1988. Guidelines on limits of exposure to radiofrequency electromagnetic fields in the frequency range from $100 \mathrm{KHz}$ to $300 \mathrm{GHz}$; Health Physics, 54, 115-123.

Kitchen, S.S. and Partridge, C.J. 1991. A review of ultraviolet radiation therapy. Physiotherapy 77(6): 423-432.

Kitchen, S. 2002. Heat and cold: conduction methods. In Kitchen, S. (ed). Electrotherapy: Evidence-based Practice, $11^{\text {th }}$ edn. Churchill Livingstone, London, pp.129-136.

Lloyd, J.J. and Evans, J.A. 1988. A calibration survey of physiotherapy equipment in North Wales. Physiotherapy 74(2): 56-61.

Low, J. 1986. Quantifying the erythema due to UVR. Physiotherapy 72: 60-64.

Low, J., Bazin, S., Docker, M., Dyson, M., Kirk, D., Kitchen, S.S., Simpson, G. 1992. Guidelines for the use of infra-red and radiant heat therapy. Physiotherapy; 499-500 (The 
Potential Hazards and Possible Safety Precautions in Electrocautery

Chartered Society of Physiotherapy- Safety of Electrotherapy Equipment Working Group).

Martin, C.J., McCallum, H.M., Heaton, B. 1990. An evaluation of radiofrequency exposure from therapeutic diathermy equipment in the light of current recommendations. Clinical Physics and Physiological Measurement 11: 53-63.

Martin, C.J., McCallum, H.M., Strelley, S., Heaton, B. 1991. Electromagnetic field from therapeutic diathermy equipment a review of hazard and precaution. Physiotherapy 77: 3-7.

McDowell, A.D., Lunt, M.J. 1992. Electromagnetic field strength measurements on megapulse units. Physiotherapy 78(4): 805809.

National Council on Radiation Protection and Measurement. 1986. Biological effects and exposure criteria for radiofrequency electromagnetic fields, NCRP report 86, Bethesda, Maryland, USA.

National Radiological Protection Board. 1989. Guidance as to restrictions on exposures to time-varying electromagnetic fields and the 1988 recommendations of the International NonIonising Radiation Committee, report NRPB GS-11, HMSO, London.

Partridge, C.J. 1992. Adverse effect of electrotherapy-preliminary report. Physiotherapy 78: 690.

Partridge, C.J. and Kitchen, S.S. 1999. Adverse effects of electrotherapy used by physiotherapists. Physiotherapy $85(6)$ : 298-303.

Quick Acne Remedy: Electric and laser therapy for skin care; www.quickacneremedy.com/natural-acn.

Repacholi, M.H. and Benwell, D.A. 91979. Using surveys of ultrasound therapy devices to draft performance standards. Health Physics 36: 679-686.

Rivest, M., Girardi, C.Q-D., Seaborne, D. and Lambert, J. 1986. Evaluation of therapeutic ultrasound devices: Performance stability over 44 weeks of clinical use. Physiotherapy Canada 39: 77-86.

Safety of Electrotherapy Equipment Working Group (SEEWG). 1990. Guidelines for the safe use of ultrasound therapy equipment. Physiotherapy 76(11): 683-684.

Schothorst, A.A., Slapper, H., Schouten, R., and Surrmond, D. 1985. UV-B doses in maintenance psoriasis phototherapy versus solar UV-B exposure. Photodermatology 2: 213-220.

Sliney, D.H. 1986. Cited in UV Radiation and Ocular Health, Proceedings of an International Roundtable Discussion, published by Cooper Vision, London.

Swanbeck, G. 1982. Ultraviolet therapy. In: Kottke, F., Stilwell, G., and Lehman J. (eds.) Krusen's Handbook of Physical Medicine and Rehabilitation ( $3^{\text {rd }}$ ed). Saunders: Philadelphia.

Ter Haar, G. 1987. Basic physics of therapeutic ultrasound. Physiotherapy 73(3): 110-112.

The Chartered Society of Physiotherapy (CSP-ERUS). 1997. Employment Relations and Union Services (ERUS): Health and Safety- Safe Practice with Electrotherapy (Shortwave Therapy). www.csp.org.uk/uploads/documents

The Health and Safety at Work Act (HSWA). 1974.

Tring, F.C. 1981. Ultraviolet light and its effects on human skin. Nursing Times 77(27): 1152-1154.

United State Patent (USP) (4124030): Electro therapeutic faradic current generator; www.freepatentsonline.com/4124030.htm accessed on 21/11/09

Urbach, F. 1982. Photocarcinogenesis. In: Regan, J.D. and Parrish, J.A. (eds). The Science of Photomedicine, Plenum Press, New York, 261-292.

Urbach, F. 1974. Ultraviolet carcinogenesis: Experimental, global and genetic aspects In: Pathak, M. (ed). Sunlight and Man, University of Tokyo Press.

Wood, K. and Reed, A. 1990. Intensity of ultraviolet radiation transmitted through a selection of screening materials. Physiotherapy 76(11): 720-724.

Young, A., Magnus, I., Davies, A., Smith, N. 1982. A comparison of phototumorigenic potential of 8-MOP and 5-MOP in hairless albino mice exposed to solar simulator radiation. British Journal of Rheumatology 108: 507-508. 